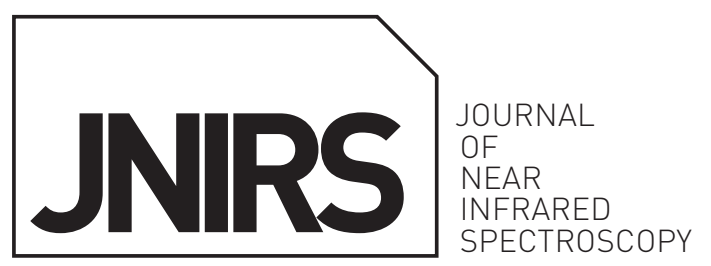

Special Issue on Medical Applications

\title{
Review
}

\section{Time-domain broadband near infrared spectroscopy of the female breast: a focused review from basic principles to future perspectives}

\author{
Antonio Pifferi, ${ }^{a, b},{ }^{*}$ Andrea Farina, ${ }^{b}$ Alessandro Torricelli, ${ }^{a}$ Giovanna Quarto, ${ }^{a}$ Rinaldo Cubeddu ${ }^{a, b}$ and \\ Paola Taronia, ${ }^{\mathrm{a}}$ \\ apolitecnico di Milano, Dipartimento di Fisica, Piazza Leonardo da Vinci 32, I-20132 Milano, Italy. E-mail: antonio.pifferi@polimi.it \\ ${ }^{b}$ Istituto di Fotonica e Nanotecnologie, Consiglio Nazionale delle Ricerche, Piazza Leonardo da Vinci 32, I-20132 Milano, Italy
}

\begin{abstract}
A review of near infrared (NIR) spectroscopy of the female breast performed using time domain broadband systems is presented, summarising the key studies performed at Politecnico di Milano. The time domain approach, based on pulsed tunable lasers and singlephoton detection, makes it possible to uncouple absorption from scattering contributions and to derive tissue optical properties in vivo non-invasively. The basic tissue absorbers in the $600-1100 \mathrm{~nm}$ range are discussed, together with the interpretation of the scattering spectra. Inter- and intra-subject variations in breast optical properties are addressed based on in vivo measurements on different women and on the same subject as a function of the menstrual cycle phase and of the measurement geometry. Results demonstrate that a specific breast type can be identified for each woman, ultimately related to the water, lipid and collagen content and to the scattering properties. As an example of the application of spectroscopy-derived information, we discuss the non-invasive optical detection of breast density as an important risk factor for breast cancer, envisaging an attractive contribution of NIR spectroscopy to the fight against breast cancer on both a medical diagnostics and research level.
\end{abstract}

Keywords: diffuse optics, diffuse spectroscopy, absorption, scattering, breast cancer, collagen, near infrared spectroscopy

\section{Introduction}

Optical biopsy li.e. the in vivo examination of biological tissues using visible and near infrared light) is an attractive tool for clinical diagnostics because of the intrinsic non-invasiveness of low-power light and the richness offered by spectral measurements providing chemical, morphological, structural and functional information on the probed medium. ${ }^{1,2}$ In the $600-1100 \mathrm{~nm}$ range, the so-called "therapeutic window", for most biological tissues the absorption coefficient $\left(\mu_{\mathrm{a}}\right)$ is relatively low $\left(<0.5 \mathrm{~cm}^{-1}\right)$ and light propagation is dominated by the reduced scattering coefficient $\left(\mu_{s}{ }^{\prime}\right)$. Thus, light can penetrate or traverse few centimetres of tissue, permitting non-invasive, in vivo investigations into the human body. In this highly diffusive regime, a wealth of methods, instruments ${ }^{2}$ and theoretical models ${ }^{3}$ have been devised to uncouple absorption from scattering contributions and to reconstruct spatial information from the visited structures smeared out by scattering interactions.

Different applications have been proposed, such as optical mammography, ${ }^{4}$ tissue oximetry, ${ }^{5}$ functional imaging of the 
brain $^{6}$ and diagnosis of osteoarticular diseases. ${ }^{7}$ More details on applications can be found in Durduran et al. ${ }^{2}$ Basic broadband spectroscopy studies, i.e. the measurement of the absorption and scattering spectra of tissues under physiological and pathological conditions, are a useful support for the onset and growing of a new application, since they can provide basic information on light penetration in the specific organ, on the optimal spectral windows offering best contrast, on the identification and role of tissue constituents and on the alterations of the absorption spectra under pathological conditions.

Time-resolved systems offer the great advantage of naturally uncoupling absorption from scattering contributions due to their different impact on the re-emitted photon temporal distribution. Thus, they are optimal tools for basic spectroscopy studies. Yet, designing a time-resolved broadband diffuse spectrometer is not a trivial task, mainly due to the need of a pulsed laser source tunable continuously over a wide spectral range and suitable detectors which will achieve a temporal resolution down to the picosecond level, together with single-photon sensitivity. The very first systems required complex laser sources and streak camera detection. ${ }^{8}$ The use of a super-continuum generated by a high-power laser widened the usable spectral range and speed of acquisition, but still required large laser systems. ${ }^{9}$ Increased sensitivity and dynamic range were attained by adopting time-correlated single-photon counting detection approaches. ${ }^{10}$ Yet, it was only recently, with the introduction of fibre-based super-continuum lasers that time domain diffuse optical spectrometers could be made compact and portable. ${ }^{11}$ Thus, in addition to basic laboratory studies, they can also be employed for use in a clinical environment.

In this paper, we will focus on diffuse optical spectroscopy of the breast by the time domain approach. Breast cancer is one of the most life-threatening pathologies in most industrialised countries. About one in eight women in the United States (12\%) will develop invasive breast cancer over the course of her lifetime. Breast cancer is by far the most common cancer in women in the United States, accounting for approximately $30 \%$ of all cases in women and breast cancer death rates are higher than those for any other cancer, besides lung cancer. Conversely, early diagnosis and consequent therapy significantly reduce mortality. ${ }^{12}$ Even small improvements in diagnosis and therapy can have a huge impact in terms of reductions in both mortality and morbidity. Breast screening essentially relies on X-ray mammography. However, false negatives and false positives hamper the efficacy of the screening and lead to unnecessary treatment, with involved physical and psychological morbidity, besides non-negligible social costs, due to the high number of treatments. Mammography is also less accurate in patients with dense glandular breasts, including young women, ${ }^{13}$ with reported sensitivity as low as $48 \% .{ }^{14}$ Other imaging modalities (for example, magnetic resonance imaging, positron emission tomography, ultrasound) offer additional diagnostic potential, yet with great social cost, or with strong dependence on the operator's experience.
Optical tools have raised much interest for the diagnosis of breast cancer due to the non-invasiveness of optical radiation and the potential low cost of photonic systems. Alongside laboratory prototypes, a number of commercial continuous wave systems have also been made available. A large number of clinical studies have risen around the use of diffuse optics for the fight against breast cancer, also fostered by support given by the European Union and USA to large multi-laboratory research projects. ${ }^{4}$ Different aspects have been investigated, such as detection and diagnosis of breast lesions with or without contrast agents, ${ }^{4,15}$ monitoring of treatment efficacy and prognosis in neoadjuvant chemotherapy ${ }^{16-21}$ and assessment of breast density as a risk factor for breast cancer. ${ }^{22-26}$ A basic knowledge of the breast optical properties through broadband spectroscopy can greatly help the optimal design of such clinical applications.

The scope of this paper is to provide an overview on broadband optical spectroscopy of the breast, focusing on timeresolved studies that were performed by our research group at the Politecnico di Milano. Previous results will be integrated with latest findings to provide a wider picture of this field, yet with a deep focusing on the specific time domain approach. In detail, we will first present the basic spectroscopy set-up and related methods, then an insight into the interpretation of absorption and scattering spectra will be given, with particular emphasis on the role of key tissue absorbers. We will show the spectral variety of breast optical properties among different subjects and discuss its implications for optical imaging. Intra-subject variations as a function of the measurement geometry and of physiological changes will be addressed to identify the range of applicability of clinical studies. Finally, an example of an application to the assessment of breast cancer risk will be presented with the latest clinical results.

\section{Materials and methods Experimental set-up}

The measurement system, developed at Politecnico di Milano, Department of Physics, is based on tunable pulsed lasers at high repetition rates $(\approx 100 \mathrm{MHz})$ as the illumination source and time correlated single-photon counting (TCSPC) electronics for detection. ${ }^{27}$ Figure 1 shows a scheme of the system set-up used in the following studies. Two different lasers can be exploited for light injection, namely an actively mode-locked Ti:sapphire laser, tunable in the 700-1100 nm range with pulses around $100 \mathrm{ps}$ (full width at half maximum, FWHM), ${ }^{28}$ or a super-continuum source with broadband emission in the $550-1700 \mathrm{~nm}$ range and pulses of a few tens of picoseconds. ${ }^{11}$ Both sources were developed in the laboratory starting from commercial lasers. In particular, a continuous wave Ti:sapphire source was modified to achieve the modelocking regime via an acousto-optic modulator, continuous operation in the whole spectral range by mirror flipping, and tuning by a Lyot filter. Conversely, the super-continuum source was coupled to a prism producing an angular dispersion of the 


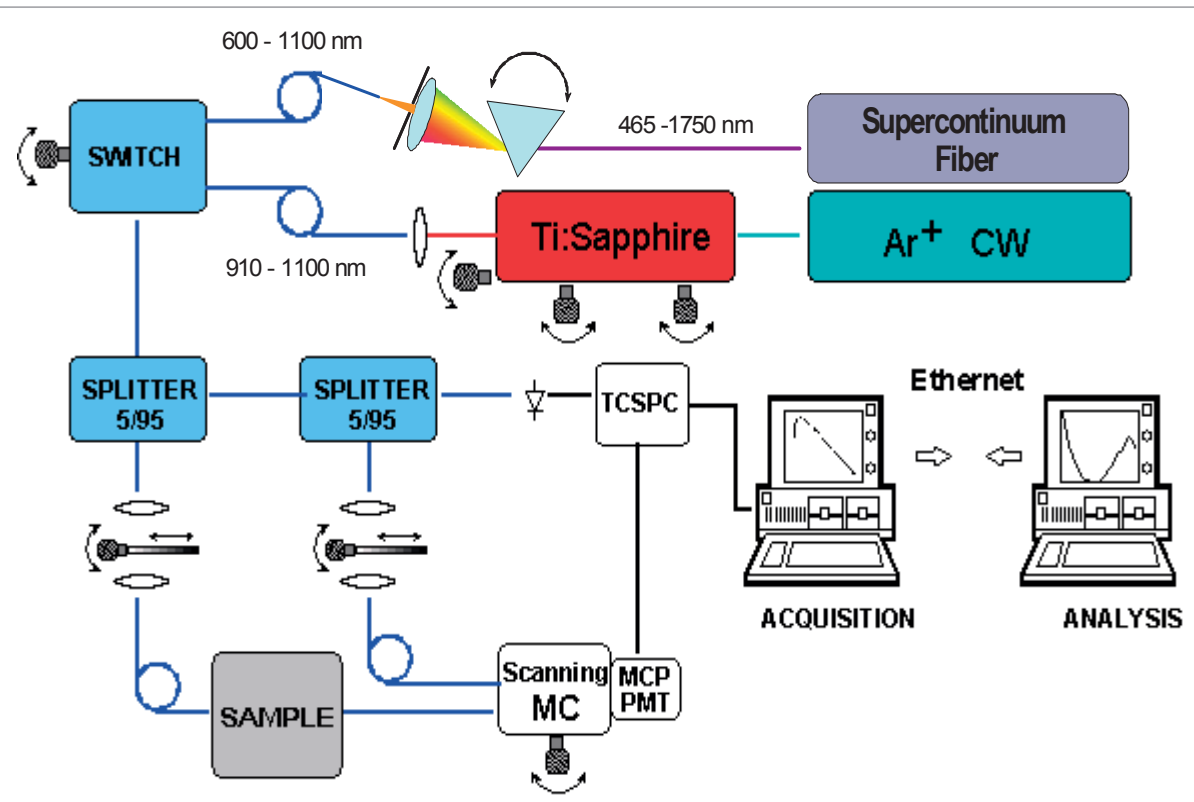

Figure 1. A scheme of the system set-up used in the current studies. Illumination is supplied either by a Mode-locked Ti:sapphire laser or by a pulsed super-continuum fibre source, with spectral selection achieved via a rotating prism. Detection is provided by timecorrelated single photon counting (TCSPC) with a temporal resolution of $\approx 70-200$ ps.

spectrum. Tuning is accomplished by focusing the dispersed light onto a slit and rotating the prism for wavelength selection, resulting in a spectral bandwidth $\approx 10 \mathrm{~nm}$ (FWHM). In both sources, a rotating circular variable attenuator filter produces an adaptable light attenuation to meet the requirements of safety regulations (a few $\mathrm{mW} \mathrm{mm}^{-2}$ ) and single-photon counting statistics (counting rate $<2-3 \%$ of the source repetition rate). These two sources present different advantages and drawbacks, with the Ti:sapphire offering high power and optimal spectral purity (FWHM $<1 \mathrm{~nm}$ and no side bands), whereas the super-continuum source covers a wide spectral range and allows smooth and easy operation.

Light is coupled into a graded index fibre for low temporal dispersion ( $50 \mu \mathrm{m}$ core) connected to a $1 \mathrm{~m}$ long, step index fibre $(1000 \mu \mathrm{m}$ core) for injection into the tissue. Re-emitted light is then harvested by a second identical step index fibre, which conveys photons to a microchannel plate photomultiplier tube (MCP-PMT) with an S1 (Ag-O-Cs) surface sensitive up to $1100 \mathrm{~nm}$. The electronic pulses are then amplified and sent to the STOP input of a TCSPC board. The synchronisation signal (START) of the board is taken either by splitting a small fraction of the Ti:sapphire beam and detecting it with a photodiode or directly from the super-continuum oscillator.

To track the effect of the system response, for each wavelength, the instrument response function is recorded by directly coupling the injection and collection fibres. A thin Teflon ${ }^{\circledR}$ foil is inserted between the fibres to mimic a Lambertian source (isotropic emission) and fill all modes in the collecting fibre. Typically, the FWHM of the instrument response function-including laser pulse width, fibre optic dispersion, PMT and electronic jitter, resolution of the TCSPC board-is in the
$70-200$ ps range, depending on the wavelength, the laser used and the operating conditions.

All operations involved in the measurement-wavelength selection, mirror flipping and cavity adjustment, adaptive attenuation, data acquisition and recording-are controlled by a PC and fully automated.

\section{Data analysis}

The measured photon temporal distribution, or temporal point spread function (TPSF), is interpreted using an analytical solution of the radiative transport equation under the diffusion approximation and applying the extrapolated boundary conditions. ${ }^{3}$ The TPSF at each wavelength is fitted with a convolution of the theoretical model with the instrument response function at the same wavelength. The free fitting parameters are the absorption, $\mu_{\mathrm{a}}$, and the reduced scattering coefficient, $\mu_{\mathrm{s}}{ }^{\prime}=\mu_{\mathrm{s}}(1-g)$, where $\mu_{\mathrm{s}}$ is the scattering coefficient and $g$ is the anisotropy factor. In the following results, according to the photon diffusion theory, only $\mu_{\mathrm{s}}{ }^{\prime}$ will be used, as that represents an effective scattering coefficient assuming isotropic scattering interactions in the medium. The fitting procedure is implemented in an ANSI-C environment (LabWindows) using the Levenberg-Marquardt algorithm. ${ }^{29}$ Since the procedure is relatively fast $(<100 \mathrm{~ms}$ per wavelength) real-time visualisation of the absorption and scattering spectra is viable while the measurement is in progress.

\section{Measurement procedures}

The breast is slightly compressed between two black PVC plates. A mild compression is adopted just to provide controlled geometry (slab) and boundaries (absorbing boundaries), and 
it is not expected to significantly affect the optical properties of breast tissue. The launching and collecting fibres are inserted into holes made through the plates-normal to their plane-so they reach the surface of the tissue. The fibres can be either placed on the same plate at a fixed inter-fibre distance ( $\rho$ ), so as to explore the tissue beneath them (reflectance), or on opposite plates on the same axis, probing the medium between them (transmittance). The fibres are positioned in the centre of the compressed breast, so they are sufficiently far from both the boundaries and the chest wall. The compressed breast thickness typically varies in the $3-7 \mathrm{~cm}$ range, while reflectance measurements are performed at inter-fibre distances of between $2 \mathrm{~cm}$ and $4 \mathrm{~cm}$.

Measurements are typically acquired from 600-700 nm up to $1000-1100 \mathrm{~nm}$ in steps of $5 \mathrm{~nm}$, averaging four acquisitions of $1 \mathrm{~s}$ each. Overall, in a given source-detector configuration, a whole spectrum is obtained in about $15 \mathrm{~min}$, including time for wavelength tuning and automatic system adjustments.

All in vivo data reported in the following were obtained from healthy subjects.

\section{Interpretation of absorption and scattering spectra}

The absorption and scattering spectra, as obtained from the fit of the TPSF, can be further analysed to derive information on the visited medium. In particular, the absorption spectrum is related to the chemical composition of the tissue through the main constituents that absorb in this spectral range.
Conversely, the scattering spectrum is linked to the fine structure of the refractive index, due to small-scale heterogeneities like cell membranes, intracellular organelles and structural fibrils. In the following, we will briefly overview the absorption properties of the key tissue constituents playing a major role in the $600-1100 \mathrm{~nm}$ range on one side and the relationship of the scattering spectrum with the microscopic structure on the other side.

\section{Absorption spectra of tissue constituents}

A good repository of the absorption spectra of some constituents in the visible-near infrared range is found in the website created by Scott Prahl. ${ }^{30}$ There is no unique information for each component since literature studies are not always in full agreement, and the absorption spectra can depend on the environment and the temperature. Moreover, some relevant spectral ranges are still missing. Thus, it is still an open field for further improvements, given the impact of an inaccurate pure substance spectrum on the estimate of tissue composition. In Figure 2, we have reported the absorption spectrum of the key tissue constituents we have considered in the following analysis, namely water, ${ }^{31}$ lipids, ${ }^{32}$ collagen, ${ }^{32}$ oxy- $\left(\mathrm{HbO}_{2}\right)$ and deoxy-haemoglobin $(\mathrm{HHb}) .{ }^{30}$ For water and lipids, the spectrum refers to the pure substance, i.e. a mass density of $1 \mathrm{~g} \mathrm{~cm}^{-3}$ and $0.9 \mathrm{~g} \mathrm{~cm}^{-3}$, respectively. $\mathrm{HbO}_{2}$ and $\mathrm{HHb}$ are referred to as molar concentration of $1 \mu \mathrm{M}$, while collagen was normalised to a reference density of $1 \mathrm{~g} \mathrm{~cm}^{-3}$.

Water is the dominant constituent in the region $>900 \mathrm{~nm}$ with a high peak around $980 \mathrm{~nm}$ and a shoulder in the $750 \mathrm{~nm}$ zone. Its relatively strong and broad absorption band is the main cause of light attenuation in tissues beyond $900 \mathrm{~nm}$.

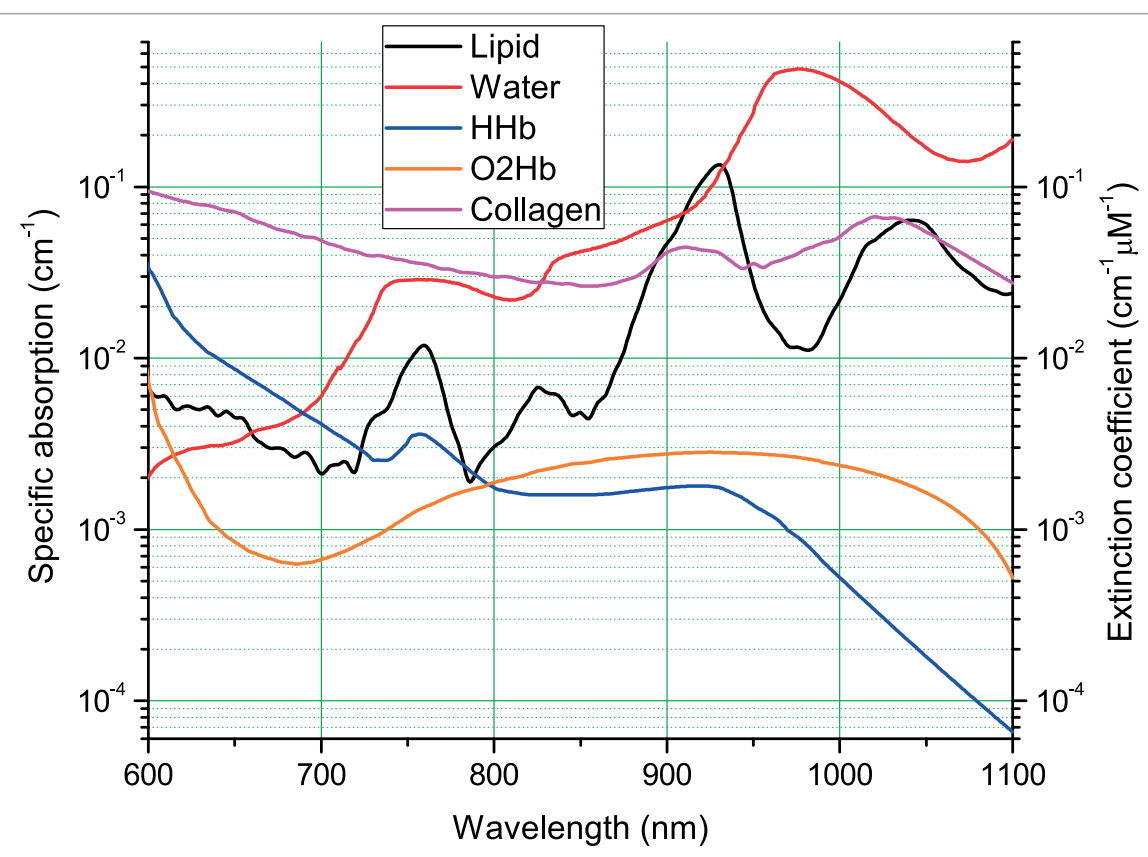

Figure 2. Absorption spectra of the major tissue absorbers: water $\left(1 \mathrm{~g} \mathrm{~cm}^{-3}\right)$, lipid $\left(0.9 \mathrm{~g} \mathrm{~cm}^{-3}\right)$, collagen $\left(1 \mathrm{~g} \mathrm{~cm}^{-3}\right)$, oxy- and deoxyhaemoglobin $\left(\mathrm{HbO}_{2}\right.$ and $\left.\mathrm{HHb}, 1 \mu \mathrm{M}\right)$. 
Lipids exhibit a narrow peak centred at $930 \mathrm{~nm}$ that exactly matches water absorption at the same wavelength (waterlipid isosbestic point). HHb shows a generally decreasing absorption upon increasing wavelength, yet with a relative maximum at $760 \mathrm{~nm}$. The steep tail in the visible red, originated by a peak at $550 \mathrm{~nm}$, causes a severe light attenuation below $650 \mathrm{~nm} . \mathrm{HbO}_{2}$, besides a decreasing tail in the red-yet less pronounced with respect to $\mathrm{HHb}$ - has a broad maximum around $900 \mathrm{~nm}$. Finally, collagen has a subtle spectrum, with a decreasing tail in the red, which can often lead to cross-talk with $\mathrm{HHb}$; a minor peak around $910 \mathrm{~nm}$ (largely masked by lipids); and a main peak at $1020 \mathrm{~nm}$, yet often overwhelmed by the high water absorption. Indeed, collagen content in breast tissue is often low $\left(<0.1 \mathrm{~g} \mathrm{~cm}^{-3}\right)$, thus its overall contribution to the absorption spectrum is limited.

As previously noticed in the literature, the absorption spectra of the main tissue constituents are not uniquely defined. As for $\mathrm{HHb}$ and $\mathrm{HbO}_{2}$ contributions, different spectra are reported, mainly in the $750 \mathrm{~nm}$ region. The version in Figure 2 is derived from Prahl's compilation, ${ }^{30}$ still lacking in information on studies beyond $1000 \mathrm{~nm} .^{33,34}$ The measurement of the $\mathrm{HHb}$ and $\mathrm{HbO}_{2}$ spectra is not trivial since haemoglobin must be derived from the lysis of red blood cells and complete deoxygenation requires a specific chemical treatment. ${ }^{33}$ Although apparently trivial, water absorption measurements performed by different researchers yielded different shapes around $980 \mathrm{~nm} .^{30,35-37}$ Furthermore, a marked dependence of the water spectrum upon temperature was observed, with appreciable variations both in amplitude and shape when moving from room to body temperature (i.e. $20^{\circ} \mathrm{C}$ to $37^{\circ} \mathrm{C}$ ). ${ }^{37}$ Moreover, a different behaviour of bound water against free water was hypothesised to lead to distinct absorption properties. ${ }^{38,39}$ The lipids spectrum reported in Figure 2 was obtained from the TRS measurement of purified pig fat. ${ }^{32}$ It is worth noting that the derivation of animal fat absorption from total attenuation continuous wave measurements (for example, via a spectrophotometer) can be severely affected by a residual scattering component, even when the fat is melted down to its liquid phase. On the other hand, the assessment of lipid absorption from transparent vegetable fat (for example seed oil) can lead to artefacts due to the important contamination of the chlorophyll contribution around $670 \mathrm{~nm} \cdot{ }^{40}$ In the case of collagen, quite a few data are available. Its contribution in biological tissues, even in cartilage, is overwhelmed by water absorption, making its characterisation awkward. It is available as a purified substance, in the form of flakes ${ }^{41}$ which, due to the high scattering contribution, can be measured only using diffuse spectroscopy techniques. It should also be remembered that different collagen types exist in nature, with the most common one, collagen type I, reported in Figure 2 but others with different abundances in diverse tissues have yet to be studied.

Knowledge of the spectral features of the key tissue constituents can be exploited to derive their abundance from a TRS measurement. Assuming a uniform distribution of the different absorbers within the probed volume, the recovered absorption spectrum of the investigated tissue can be fitted with a linear combination of the reference components' spectra to retrieve their concentration using Beer's law:

$$
\mu_{\mathrm{a}}(\lambda)=\sum_{i=1}^{N} c_{i} \varepsilon_{i}(\lambda)
$$

where $c_{i}$ is the concentration of the $i$-th absorber and $\varepsilon_{i}(\lambda)$ its extinction spectrum.

\section{Scattering spectra}

The interpretation of the scattering spectrum is not as straightforward. In principle, the scattering properties of a tissue could be derived from the study of the propagation of the electromagnetic field within a locally inhomogeneous medium. In practice, the complexity of the model, the huge number of unknowns and the complex geometry of scattering centres make it unfeasible to treat the problem rigorously. Most of all, the microscopic heterogeneity of tissue causes a smearing of the fine spectral details when a bulk measurement is considered, resulting in a generally smooth and monotonically decreasing scattering spectrum.

Thus, an empirical macroscopic description is usually adopted. It was observed, both from simulations using the Mie scattering model and from experimental measurements, that the scattering spectrum in most biological tissues in the "therapeutic window" roughly follows a power law in the form: 42,43

$$
\mu_{s}^{\prime}(\lambda) \approx a\left(\frac{\lambda}{\lambda_{0}}\right)^{-b}
$$

where $\lambda_{0}$ is an arbitrary reference wavelength used for dimensional requirements, $b$ is often termed the scattering power and determines the slope of the spectrum in a log-log scale, while $a$ is an amplitude factor. Incidentally, a represents the reduced scattering coefficient extrapolated at $\lambda_{0}$. A useful physical meaning of $b$ can be hinted at by analysing a set of Mie simulations for homogeneous spheres with different radii using Equation (2). In this case, $b$ can be related to the radius (dimension) of the sphere, while $a$ is related to the volume concentration. ${ }^{43}$ Thus, $a$ and $b$ derived for a biological tissue can be converted to an effective concentration and dimension of the scattering centres. It must be underlined that these figures are not the "real" average size and density of the tissue scatterers, but rather effective parameters for a sphere distribution yielding an equivalent scattering spectrum. Nonetheless, these empirical quantities can be profitably used to quantify microstructure-related features and possibly classify different tissue structures.

Equations (1) and (2) can also be exploited in a different way. Instead of fitting the TPSF individually at each wavelength to recover the absorption and scattering spectra, the whole set of TPSFs, at all wavelengths, can be globally fitted with a diffusion equation model where the absorption and reduced scattering coefficients are linked [through Equation (1) and Equation (2)] to the absorber concentrations and the structural $a$ and $b$ parameters. ${ }^{44}$ This a priori condition greatly reduces the number of unknowns and improves the robustness of the fit 
at low signal levels. The drawback is that the spectra of the components must be known in advance and it is not possible to directly identify some hidden missing components or spectral alterations in the primitive absorption spectra. Also, the single exponent component in the scattering spectrum might be an oversimplification in real tissues, where bi-modal distributions could be identified.

\section{In vivo breast spectroscopy Inter-subject variations}

The variety of breast optical properties that can be seen among different women is represented in Figure 3. The in vivo spectra of the absorption [Figure 3(a)], the reduced scattering [Figure $3(b)$ ] and the effective attenuation coefficient [Figure $3(c)]$ are presented for 10 healthy subjects lage range 26-63 years). Data were obtained averaging the optical properties measured on both breasts in transmittance geometry right in the central region, with the breast being slightly compressed between two black PVC plates (thickness range of compressed breast $2.9-6.3 \mathrm{~cm}$, median $4.0 \mathrm{~cm}) .{ }^{32}$ The absorption spectrum [Figure 3(a)] shows large variations both in wavelength and among subjects. The main spectral features that can be easily identified are the decreasing tail of $\mathrm{HHb}$ and $\mathrm{HbO}_{2}$ in the red, the lipid peak at $930 \mathrm{~nm}$ and the water absorption maximum around $980 \mathrm{~nm}$. The region around $760 \mathrm{~nm}$ is quite complex due to the overlapping of minor water, lipid and $\mathrm{HHb}$ peaks. The broad absorption band of $\mathrm{HbO}_{2}$ at about $900 \mathrm{~nm}$ can not be identified since it is overwhelmed by the marked water and lipid peaks; conversely, it contributes to the overall absorption in the NIR region. Also, collagen features are not visible since the intrinsic absorption of collagen is rather low, thus making its accurate quantification often non-trivial. The key differences among women can easily be seen in the 900-1000 nm region where different abundances of lipids and water yield substantially diverse absorption properties. It is worth noting that, at $930 \mathrm{~nm}$ (the water-lipid isosbestic point), all subjects show a similar absorption, not surprisingly since at this wavelength water and lipids are the main constituents, thus breast absorption will depend mainly on the water+lipid total content (accounting for almost $100 \%$ of the total volume) and not on their relative abundance.

The scattering spectra [Figure 3(b)] exhibit lower variations. At $800 \mathrm{~nm} \mu_{\mathrm{s}}^{\prime}$ ranges between $10 \mathrm{~cm}^{-1}$ and $17 \mathrm{~cm}^{-1}$ for all subjects. The spectra can vary both in amplitude and in slope, the latter being generally higher in water-rich breasts. The reason is that the breast with a prevalence of glandular and fibrous tissue has an important water content compared to a more adipose breast and also, due to the presence of collagen fibrils, a steeper descendent scattering spectrum.

The knowledge of both $\mu_{\mathrm{a}}$ and $\mu_{\mathrm{s}}{ }^{\prime}$ can be used to derive an effective attenuation coefficient $\mu_{\text {eff }}=\sqrt{3 \mu_{\mathrm{a}} \mu_{\mathrm{s}}{ }^{\prime}}$, which yields the total light attenuation while travelling through the medium. ${ }^{45}$ As a rough approximation, light injected as a collimated pencil beam of intensity $I_{0}$ into a homogeneous slab of thickness $d$ (for example, the breast compressed between two parallel plates) is re-emitted on the opposite side along the axis with an intensity $I \propto I_{0} \exp \left(-\mu_{\text {eff }} d\right) .{ }^{45}$ Using the absorption and scattering spectra shown above, the effective attenuation spectra [Figure 3(c)] can be derived for the 10 subjects. Again, large spectral and inter-subject variations are observed. The region between $650 \mathrm{~nm}$ and $850 \mathrm{~nm}$ shows the lowest attenuation and, indeed, the vast majority of studies involving breast imaging and tomography have explored that spectral range. Conversely,

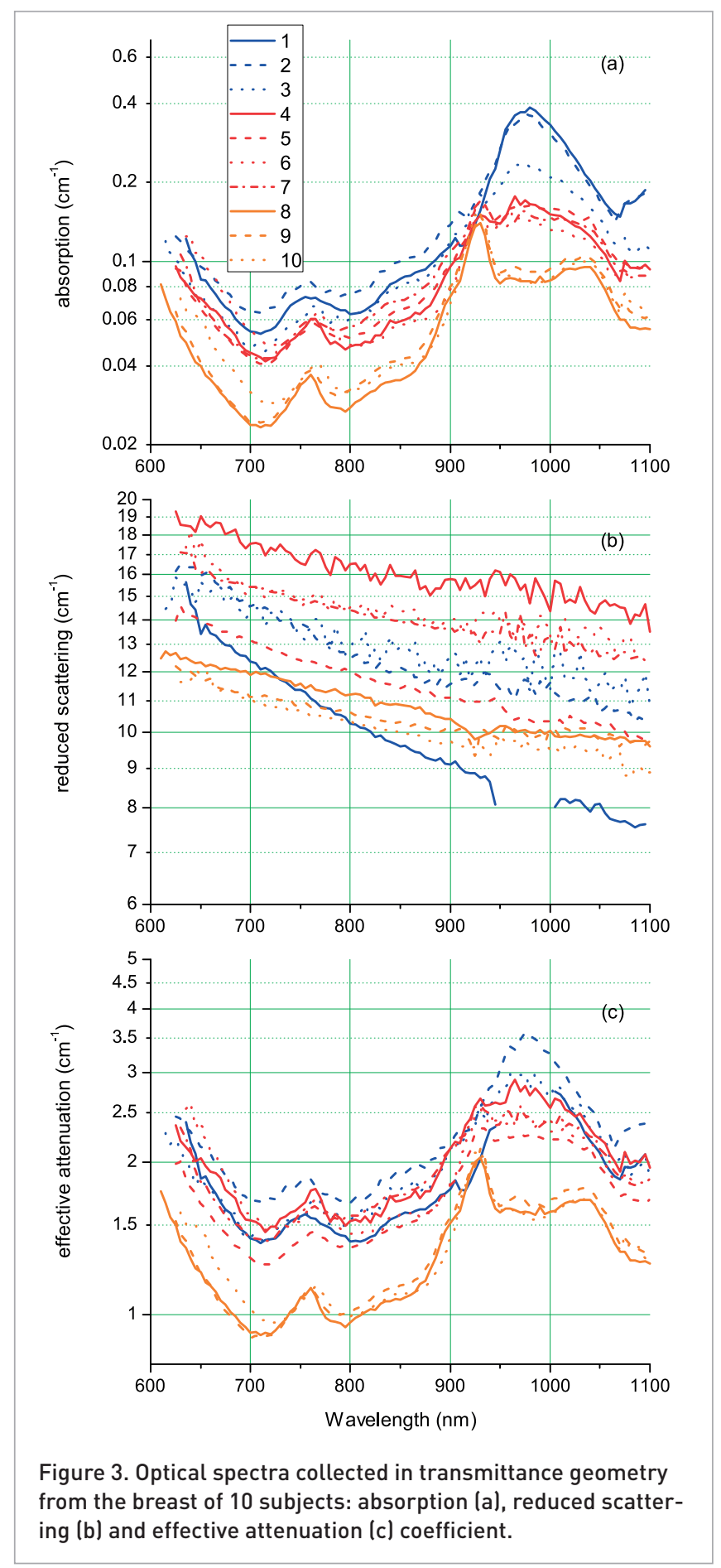


the region around $1100 \mathrm{~nm}$ is largely unexplored, despite light penetration being comparable to the $650-850 \mathrm{~nm}$ range and the main tissue absorbers show quite distinct spectral features. Clearly, beyond 900-1000 nm, detectors are noisier and less efficient, although, also for time-resolved measurements, InGaAs single-photon detectors are reaching quite useful performances. ${ }^{46}$

The absorption and scattering spectra can be further analysed to derive tissue composition and structure, as described in the section "Scattering spectra" and as shown in Figure 4. The concentrations of the main tissue absorbers in this spectral range (water, lipids, collagen, $\mathrm{HHb}, \mathrm{HbO}_{2}$ ) for the 10 volunteers are shown in Figure 4(a), where subjects are ordered according to decreasing water content. Clearly, upon decreasing water, lipid abundance increases. In contrast, collagen is a main structural protein in fibrous-glandular tissue, which is also rich in water. Thus, the amounts of collagen and water are positively correlated. $\mathrm{HbO}_{2}$ and $\mathrm{HHb}$ contents are in the $7-20 \mu \mathrm{M}$ and $2-10 \mu \mathrm{M}$ range, respectively. The corresponding $a$ and $b$ values derived from an empirical Mie model of scattering spectra are shown in Figure 4(b) for the same subjects in the same order. Higher $b$ values, implying smaller scattering centres, are seen in water-rich breasts and are indicative of more fibrous tissue.

\section{Effect of measurement geometry}

The effect of the measurement geometry on the retrieved absorption spectrum is shown in Figure 5 for two healthy premenopausal subjects (\#1, 40 years and \#2 50 years). Reflectance measurements at source-detector distances of $2 \mathrm{~cm}, 3 \mathrm{~cm}$ and $4 \mathrm{~cm}$ are shown, together with transmittance measurements along the axis, always with the breast slightly compressed between two black PVC plates (thickness $3.1 \mathrm{~cm}$ and $4.3 \mathrm{~cm}$ for subjects \#1 and \#2, respectively)..$^{47}$ With regards to the absorption spectra [Figure 5(a)], the water peak is more prominent for reflectance measurements at larger inter-fibre distances, whereas transmittance measurements are more similar to reflectance at $2 \mathrm{~cm}$. A similar trend is also observed in other subjects, while transmittance measurements can yield a water peak intermediate compared to reflectance spectra (data not shown). This is in agreement with the uneven distribution of the mammary gland, denser in the centre of the breast, and covered by fatty tissues. In the case of reflectance measurements, the maximum depth sensitivity increases upon increasing the photon transit time. Thus, larger inter-fibre distances are often more sensitive to the mammary gland and detect a higher water content. Conversely, transmittance measurements probe the whole thickness and are thus possibly more representative of the average breast properties. The scattering spectra [Figure 5(b)] show larger dependence on the measurement geometry, with changes in amplitude and, in the case of Subject \#2, also in slope.

\section{Physiological changes}

As for other imaging and diagnostics modalities (for example ultrasound, $X$-rayl, an issue is the potential variation of the

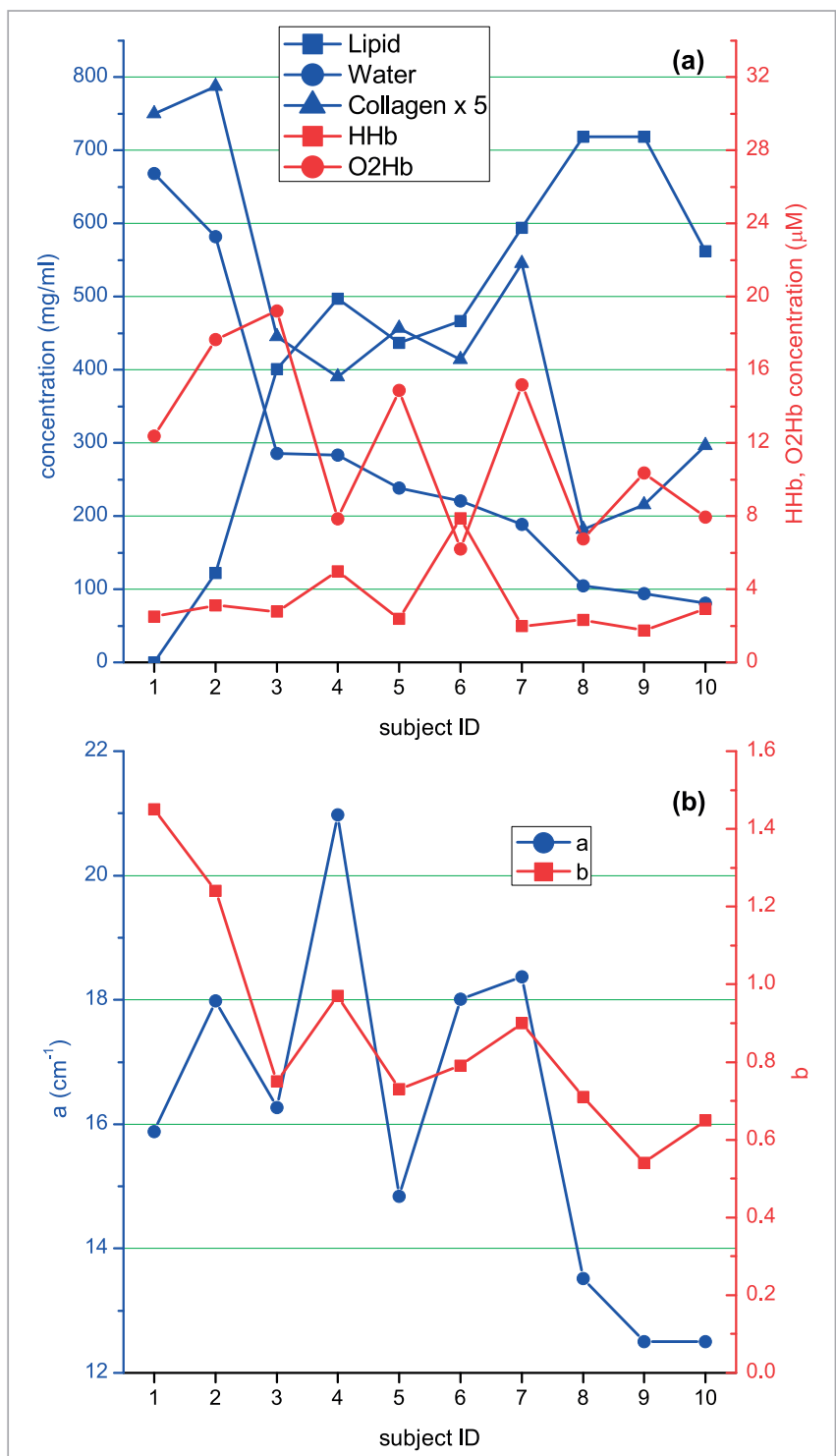

Figure 4. Tissue composition in terms of water, lipid, collagen, $\mathrm{HbO}_{2}$ and $\mathrm{HHb}(\mathrm{a}$ ) and scattering parameters (b) for the 10 subjects shown in Figure 3 . Increasing subject identification number corresponds to decreasing water content.

measurements over time, particularly related to the physiological changes within the breast. This aspect is particularly relevant for comparing breast measurements on different women. In line with this, we tracked the breast absorption and scattering spectrum on a healthy subject (age 36) over two months, with measurements performed every four to five days. ${ }^{48}$ The spectra were then grouped according to the phase of the menstrual cycle, as defined in Table $1 .{ }^{49}$ Figure 6 shows the absorption and scattering spectra in transmittance geometry, averaged over the cycle phases. The absorption spectrum [Figure 6(a)] shows very little variation, apart from some increase in water content in phases IV and $V$, which can be explained because of the water retention leading to higher water concentration. 


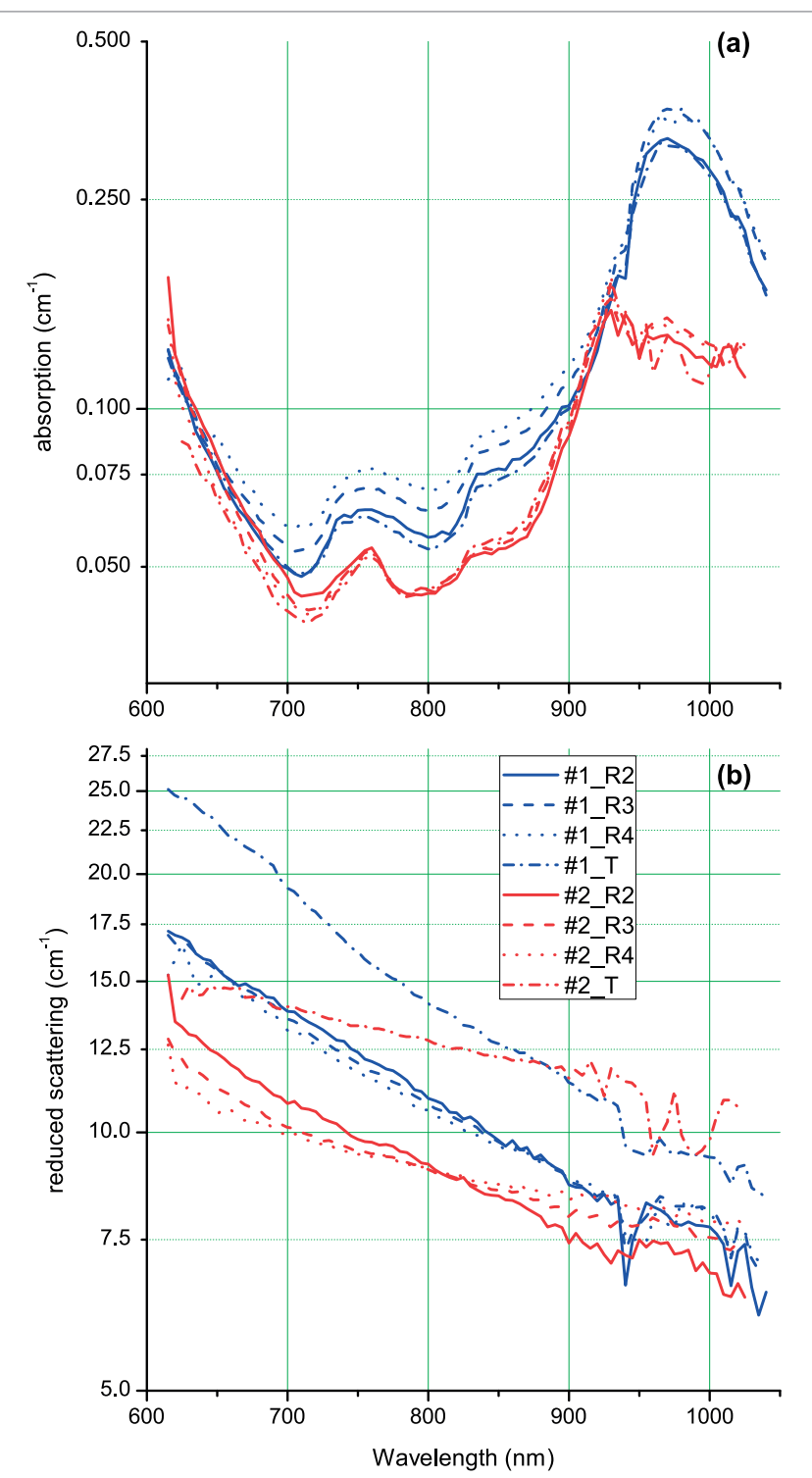

Figure 5. Absorption (a) and reduced scattering (b) spectra obtained in transmittance geometry $(T)$ or in reflectance geometry with inter-fibre distance of $2 \mathrm{~cm}$ (R2), $3 \mathrm{~cm}$ (R3), or $4 \mathrm{~cm}$ (R4) for two subjects (for example \#1_R2 refers to spectra obtained from Subject \#1 in reflectance geometry with interfibre distance of $2 \mathrm{~cm}$ ).

The scattering spectrum [Figure $6(b)$ ] shows somewhat larger variations, with higher scattering values during phases
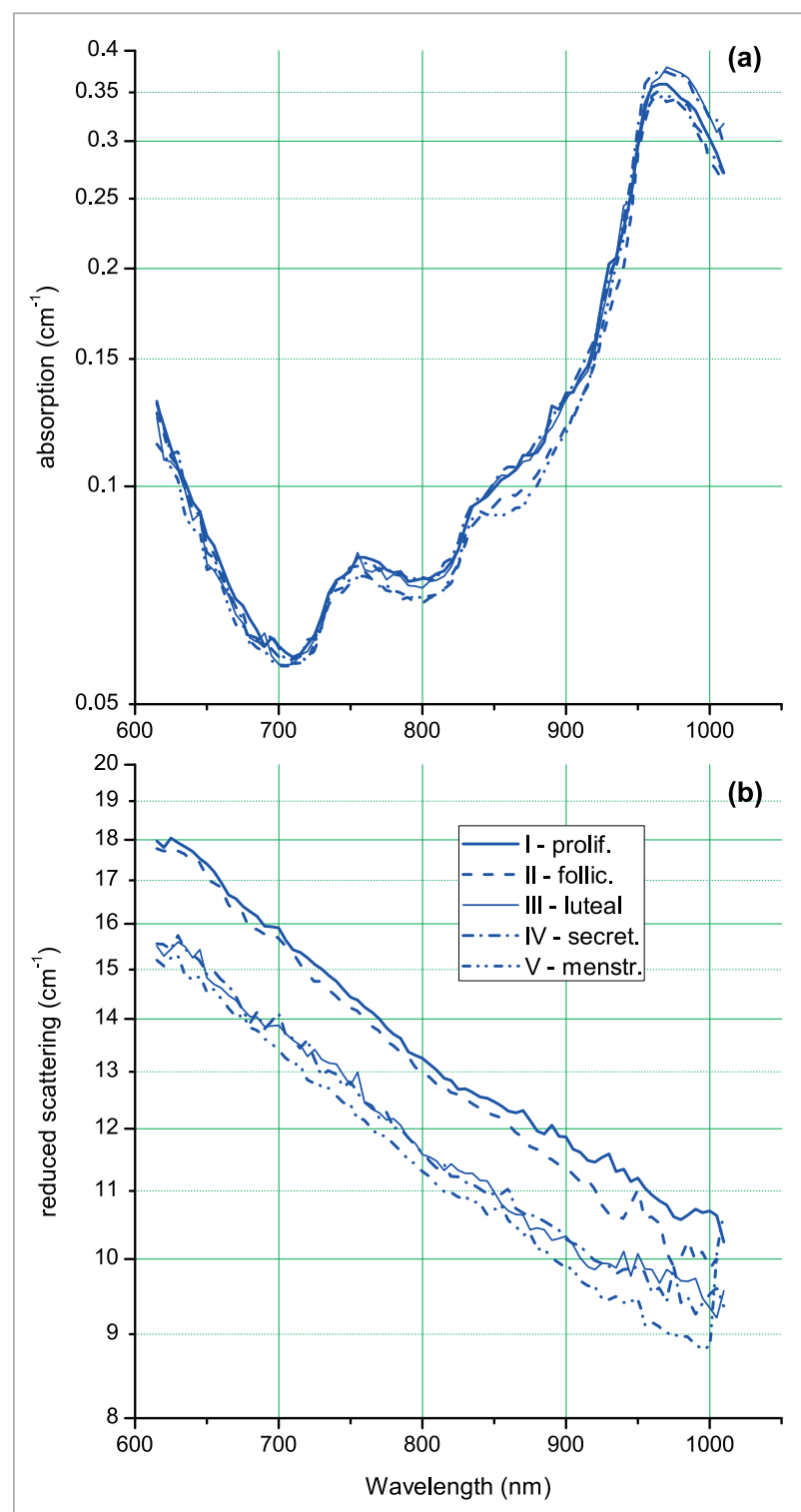

Figure 6. Absorption (a) and reduced scattering (b) spectra obtained from a subject in the different phases of the menstrual cycle (see Table 1).

Table 1. Definition of menstrual cycle phases laccording to Vogel et al. ${ }^{49}$ ).

\begin{tabular}{|c|l|l|l|}
\hline Phase number & Phase name & Days & Structure \\
\hline I & Proliferative & $3-7$ & Dense cellular stroma \\
\hline II & Follicular & $8-14$ & Dense cellular-collagenous stroma \\
\hline III & Luteal & $15-20$ & Loose broken stroma \\
\hline IV & Secretory & $21-27$ & Loose oedematous stroma \\
\hline V & Menstrual & $28-2$ & Dense cellular stroma \\
\hline
\end{tabular}

I and II, possibly related to the changes in structure occurring in the mammary gland (see Table 1). In any case, the slope 
of the spectrum is largely stable all over the phases. More specifically, changes in the absorption and scattering properties that are related to the menstrual cycle are smaller than differences observed among subjects with different breast type (Figure 4 compared to Figure 5). Thus, a classification of breast type based on the average optical properties is feasible with a large tolerance on the timing of the examination and longitudinal studies on the evolution of breast optical properties (for example, following neoadjuvant chemotherapy) ${ }^{16-20}$ are also feasible.

It is worth mentioning here that time-resolved diffuse spectroscopy provides absolute values of $\mu_{\mathrm{a}}$ and $\mu_{\mathrm{s}}{ }^{\prime}$ without the need of calibration or a priori modelling of the optical spectra. Also, since the retrieval of $\mu_{\mathrm{a}}$ and $\mu_{\mathrm{s}}{ }^{\prime}$ depends on the temporal shape of the photon distribution and not on its amplitude, the measurement is completely unaffected by optical coupling or skin pigmentation. This leads to a greater robustness of the technique and entitles one to use absolute thresholds for breast or lesion classification.

\section{Breast cancer risk prediction}

As an example of the potential of breast spectroscopy for the fight against breast cancer, we present the results on an ongoing clinical study for the non-invasive assessment of breast cancer risk by diffuse optical spectroscopy. ${ }^{22}$ The goal is to ascertain if optical measurements can be effectively used to derive the breast density (defined by X-ray mammography), which is an important risk factor for the onset of breast cancer. ${ }^{50}$ Details on the system set-up, on the clinical procedures, and on data analysis are given in a report on the preliminary results of the study. ${ }^{22}$ Here, we just summarise those preliminary results that were obtained on the first 49 women lage range 31-78 years, median 51) and confirmed later on a higher number of subjects (71 subjects, data not published). Briefly, the clinical system is operated at seven discrete wavelengths $1635 \mathrm{~nm}, 685 \mathrm{~nm}$, $785 \mathrm{~nm}, 905 \mathrm{~nm}, 930 \mathrm{~nm}, 975 \mathrm{~nm}, 1060 \mathrm{~nm}$ ) and an XY translation stage makes it possible to scan the whole breast in transmittance geometry while softly compressed between two transparent glass plates (median thickness of the compressed breast $5.2 \mathrm{~cm}$ ). The scan time depends on the size of the scanned area, ranging typically from 5 min to 10 min per image. The optical properties averaged over the whole scanned area, excluding the borders that might be affected by artefacts, are used to retrieve the average tissue composition $\left(\mathrm{HHb}, \mathrm{HbO}_{2}\right.$, water, lipids, collagen) and structure $(a, b)$ of the breast. Mammographic breast density was graded applying the Breast Imaging and Reporting Data System (BI-RADS), which classifies breasts as: (i) almost entirely fat (category 1); (ii) scattered fibroglandular densities (category 2); (iii) heterogeneously dense (category 3); and (iv) extremely dense (category 4).

Figure 7 shows the correlation of the different optical parameters with the BI-RADS categories. Since X-ray attenuation is mainly due to water and also to glandular and stromal tissues, a strong correlation is observed between breast density and different optically retrieved parameters (water, collagen, $b$ and, inversely, lipids). The correlation is even stronger using the synthetic optical index, Ol defined as:

$$
\text { OI }=\frac{[\text { water }][\text { collagen }] \times b}{[\text { lipid }]}
$$

where parameters that are expected to increase with breast density (i.e. the concentrations of water and collagen, and the scattering slope $b$ ) are all multiplied and divided by lipid
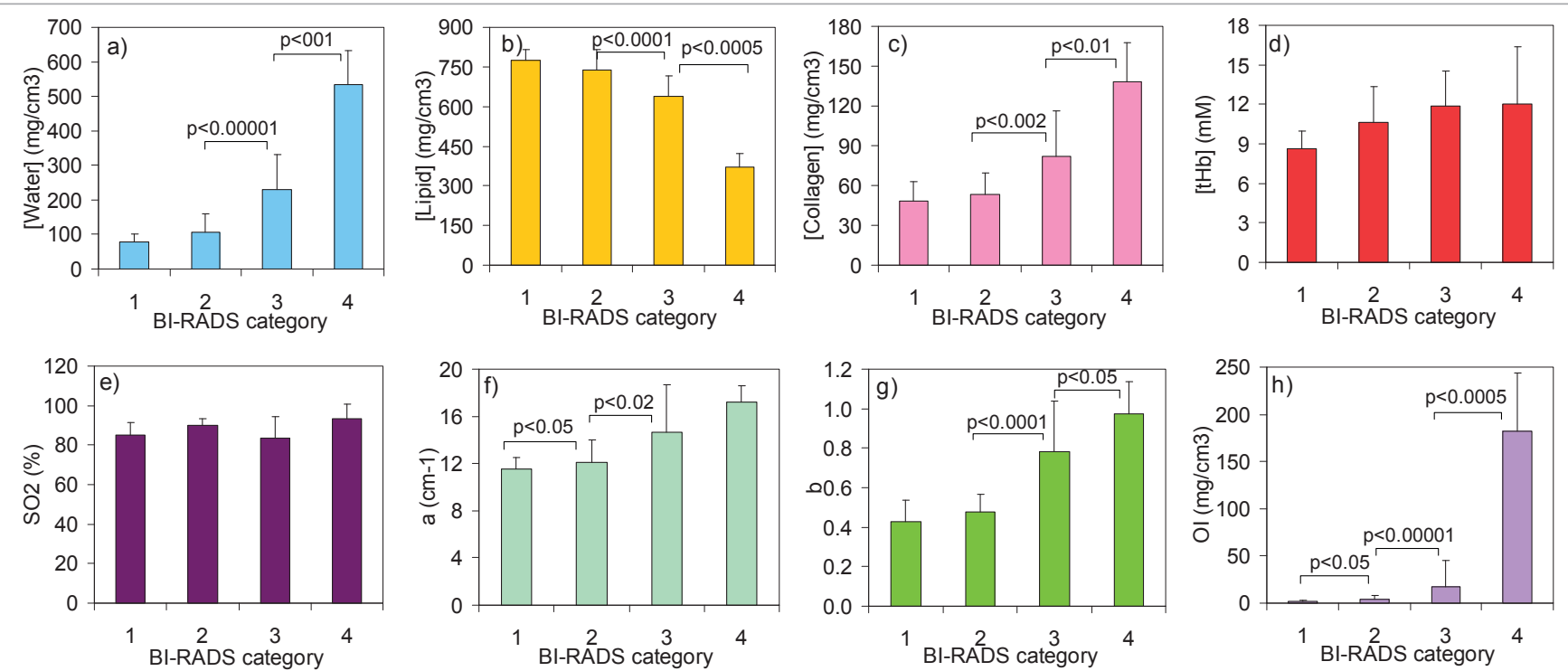

Figure 7. Optically derived parameters as a function of BI-RADS categories: water (a), lipid (b), collagen (c), tHb (d), $\mathrm{SO}_{2}(\mathrm{e}), a$ (f), $b$ (g), and 0 ( $(h)$. Results are expressed as mean $\pm S D$. Statistical significance of the difference between different categories was estimated with the Mann-Whitney test. $P$ values $<0.05$ were considered significant and are reported in the figure. (Reproduced, with permission, from Taroni et al. ${ }^{22}$. 
concentration, which conversely is expected to decrease upon increasing breast density.

Overall, the optical index can significantly separate category 4 , the highest density (highest cancer risk), from category 3 ( $P$ $<0.0001$ l) and category 4 from all the others with even higher confidence. Comparable conclusions were drawn in another large clinical study based on diffuse transmittance spectroscopy yet with continuous wave acquisition. ${ }^{23}$ Instead of extraction of tissue constituent concentrations, the study treated the whole spectra with principal component analysis, deriving a good separation of breast density classes using statistically inferred spectral features.

All these findings indicate a fascinating perspective. Breast cancer is impressively impacted by early diagnosis. If the tumour is diagnosed in the early stage (nodule $<1 \mathrm{~cm}$, and no lymph node involvement), complete cure is gained in $95 \%$ of cases. Thus, optical tools could be used to identify a restricted cluster of women at higher risk for whom a personalised diagnostic path (for example, recurrent clinical examination, ultrasound, magnetic resonance imaging, prevention) could be foreseen and to hit cancer at its early onset or even prevent it. Since light at such a low power is completely safe, screening can be performed at a young age, different from X-ray mammography, in order to also address young women, in whom tumours are often aggressive and with even harsher impact on life quality and society. Further, quite recently, breast density was found to correlate more strongly with some specific tumour types, generally with higher index of malignancy. ${ }^{51,52}$ Thus, knowledge of breast classification can help clinicians to set up an optimised diagnostic strategy. So far, only the direct correlation of optical parameters to mammographic breast density has been considered. Yet, the direct correlation of single parameters, such as collagen content or $b$ value (related to microstructure) to cancer risk could prove valuable. Indeed, it has been hinted that collagen itself is directly related to breast carcinogenesis. ${ }^{53-55}$ Thus, its detection could lead to a better demarcation of risk clusters, with better affordability for society and more targeted interventions. Last, but not least, easy non-invasive in vivo assessment of collagen and structure can greatly benefit clinical research, trying to elucidate the aetiology of breast oncogenesis with respect to the proven correlation with breast density.

\section{Conclusions}

In conclusion, we have presented an overview of in vivo breast spectroscopy using time-resolved broadband diffuse optical spectroscopy, in view of the potential use of optical tools for diagnosis and treatment monitoring of breast cancer. The key advance of the time-resolved approach is the capability to uncouple absorption from scattering contributions, leading to the estimate of tissue absorption and scattering spectra with no need for external calibration or statistical gauging on a large population. Working in the $600-1100 \mathrm{~nm}$ range, the main spectral features related to $\mathrm{HHb}, \mathrm{HbO}_{2}$, water, lipids and collagen can be identified from the absorption spectrum, together with tissue-structure parameters from the scattering spectrum. A large variability in tissue composition and structure is observed among different women, leading to the classification of different breast types, namely mainly lipid patterns on the one hand and fibrous and water rich patterns on the other. Due to the heterogeneous structure of the breast, reflectance measurements can yield different results for increasing inter-fibre distances because of larger depth sensitivities for longer distances. Conversely, transmittance measurements provide an average picture through the whole breast, and could be preferable to derive average information. Intra-subject physiological variations arising from different phases of the menstrual cycle are much smaller than inter-subject differences, thus allowing one to perform intersubject classification and grading and longitudinal studies on the same women, for example for treatment monitoring. After presenting the basic knowledge of physiological breast optical properties, an attractive use of optical tools for non-invasive assessment of breast cancer risk was presented.

Despite a large number of studies on breast imaging and spectroscopy, the field is still quite open both for new research directions, as tumour detection with contrast agents, ${ }^{15}$ dynamic breast imaging, ${ }^{56}$ assessment of tumour-related spectral alterations, ${ }^{39}$ monitoring of neoadjuvant chemotherapy, ${ }_{1}^{18}$ assessment of cancer risk ${ }^{22}$ and also for novel technical approaches, such as exploring the spectral range well beyond $1000 \mathrm{~nm}$, or exploiting dense high-throughput sourcedetector arrangements.

Time-resolved diffuse optical spectroscopy has particularly benefited from the impressive growth of photonics during the last decades. What once required ultra-fast laser laboratories hosted at large-scale facilities ${ }^{9}$ can now be squeezed into portable clinical prototypes. ${ }^{11}$ Furthermore, research is moving at an impressive pace, mainly towards miniaturisation, integration, multiplexing and cost reduction. Novel detection schemes are being proposed, making feasible new approaches that were just unthinkable, such as deep tissue spectroscopy using a null inter-fibre distance. ${ }^{57}$ The real clue now seems to be the identification of clear endpoints that are meaningful for physicians. To this end, basic spectroscopy studies can still play an important role.

\section{Acknowledgements}

The research leading to these results received partial funding from the European Community's Seventh Framework Programme under grant agreement n.228334 (LASERLAB-EUROPE).

\section{References}

1. A. Yodh and B. Chance, "Spectroscopy and imaging with diffusing light", Phys. Today 48, 34 (1995). doi: $\underline{10.1063 / 1.881445}$ 
2. T. Durduran, R. Choe, W.B. Baker and A.G. Yodh, “Diffuse optics for tissue monitoring and tomography", Rep. Prog. Phys. 076701 (2010). doi: 10.1088/0034-4885/73/7/076701

3. F. Martelli, "ABC of near infrared photon migration in tissues: the diffusive regime of propagation", J. Near Infrared Spectrosc. 20, 29 (2012). doi: 10.1255/jnirs.974

4. D.R. Leff, O.J. Warren, L.C. Enfield, A. Gibson, T. Athanasiou, D.K. Patten, J. Hebden, G.Z. Yang and A. Darzi, "Diffuse optical imaging of the healthy and diseased breast?: A systematic review", Breast Cancer Res. Treat. 108, 9 (2008). doi: 10.1007/s10549-007-9582-z

5. M. Ferrari and V. Quaresima, "Near infrared brain and muscle oximetry, from the discovery to current applications", J. Near Infrared Spectrosc. 20, 1 (2012). doi: 10/1255/jnirs.973

6. D. Contini, L. Zucchelli, L. Spinelli, M. Caffini, R. Re, A. Pifferi, R. Cubeddu and A. Torricelli, "Brain and muscle near infrared spectroscopy/imaging techniques”, J. Near Infrared Spectrosc. 20, 15 (2012). doi: 10.1255/jnirs.977

7. A.H. Hielscher, H.K. Kim, L.D. Montejo, S. Blaschke, U.J. Netz, P.A. Zwaka, G. Illing, G.A. Muller and J. Beuthan, "Frequency-domain optical tomographic imaging of arthritic finger joints", IEEE Trans. Med. Im. 10, 1725 (2011). doi: 10.1109/TMI.2011.2135374

8. M. Ferrari, Q. Wei, L. Carraresi, R.A. De Blasi and G. Zaccanti, "Time-resolved spectroscopy of human forearm”, J. Photochem. Photobiol. B 16, 141 (1992). doi: 10.1016/1011-1344(92).80005-G

9. S. Andersson-Engels, R. Berg, A. Persson and S. Svanberg, "Multispectral tissue characterization with time-resolved detection of diffusely scattered white light", Opt. Lett. 18, 1697 (1993). doi: $\underline{10.1364 /}$ OL.18.001697

10. R. Cubeddu, A. Pifferi, P. Taroni, A. Torricelli and G. Valentini, "Noninvasive absorption and scattering spectroscopy of bulk diffusive media?: An application to the optical characterization of human breast", Appl. Phys. Lett. 74, 874 (1999). doi: 10.1063/1.123395

11. A. Bassi, J. Swartling, C.D. Andrea, A. Pifferi, A. Torricelli and R. Cubeddu, "Time-resolved spectrophotometer for turbid media based on supercontinuum generation in a photonic crystal fiber", Opt. Lett. 29, 2405 (2004). doi: 10.1364/OL.29.002405

12. L. Tabar, M.-F. Yen, B. Vitak, H.-H.T. Chen, R.A. Smith and S.W. Duffy, "Mammography service screening and mortality in breast cancer patients?: 20-year follow-up before and after introduction of screening", Lancet $\mathbf{3 6 1}$, 1405 (2003). doi: 10.1016/S0140-6736(03).13143-1

13. E. Marshall, "Brawling over mammography”, Science 327, 936 (2010). doi: 10.1126/science.327.5968.936

14. T.M. Kolb, J. Lichy and J.H. Newhouse, "Comparison of the performance of screening mammography, physical examination and breast US and evaluation of factors that influence them: An analysis of 27,825 patient evaluations", Radiology 225, 165 (2002). doi: $10.1148 /$ radiol.2251011667
15. D. Grosenick, H. Wabnitz and B. Ebert, "Recent advances in contrast-enhanced diffuse optical imaging of diseases using indocyanine green", J. Near Infrared Spectrosc. 20, 203 (2012). doi: 10.1255/inirs.964

16. A. Cerussi, D. Hsiang, N. Shah, R. Mehta, A. Durkin, J. Butler and B.J. Tromberg, "Predicting response to breast cancer neoadjuvant chemotherapy using diffuse optical spectroscopy", Proc. Natl. Acad. Sci. USA 104, 4014 (2007). doi: 10.1073/pnas.0611058104

17. D. Roblyer, S. Ueda, A. Cerussi, W. Tanamai, A. Durkin, R. Mehta, D. Hsiang, J. Butler, C. McLaren, W.P. Chen and B. Tranberg, "Optical imaging of breast cancer oxyhemoglobin flare correlates with neoadjuvant chemotherapy response one day after starting treatment", Proc. Natl. Acad. Sci. USA 108, 14626 (2011). doi: $10.1073 /$ pnas.1013103108

18. M.G.G. Pakalniskis, W.A.A. Wells, M.C.C. Schwab, H.M.M. Froehlich, S. Jiang, Z. Li, T.D.D. Tosteson, S.P.P. Poplack, P.A.A. Kaufman, B.W. Poque and K.D. Paulsen, "Tumor angiogenesis change estimated by using diffuse optical spectroscopic tomography: demonstrated correlation in women undergoing neoadjuvant chemotherapy for invasive breast cancer?", Radiology 259, 365 (2011). doi: 10.1148/radiol.11100699

19. H. Soliman, A. Gunasekara, M. Rycroft, J. Zubovits, R. Dent, S. Jacqueline, M.J. Yaffe, G.J. Czarnota and J. Spayne, "Functional imaging using diffuse optical spectroscopy of neoadjuvant chemotherapy response in women with locally advanced breast cancer", Clin. Cancer Res. 16, 2605 (2010). doi: 10.1158/1078-0432.CCR09-1510

20. Q. Zhu, S. Tannenbaum, P. Hegde, M. Kane, C. Xu and S.H. Kurtzman, "Noninvasive monitoring of breast cancer during neoadjuvant chemotherapy using optical tomography," Neoplasia 10, 1028 (2008). doi: 10.1593/ neo.08602

21. L.C. Enfield, G. Cantanhede, D. Westbroek, M. Douek, A.D. Purushotham, J.C. Hebden and A.P. Gibson,

"Monitoring the response to primary medical therapy for breast cancer using three-dimensional time-resolved optical mammography", Technol. Cancer Res. Treat. 10, 533 (2011).

22. P. Taroni, A. Pifferi, G. Quarto, L. Spinelli, A. Torricelli, F. Abbate, A. Villa, N. Balestreri, S. Menna, E. Cassano and R. Cubedda, "Noninvasive assessment of breast cancer risk using time-resolved diffuse optical spectroscopy", J. Biomed. Opt. 15, 15 (2010). doi: 10.1117/1.3506043

23. K.M. Blackmore, J.A. Knight and L. Lilge, "Association between transillumination breast spectroscopy and quantitative mammographic features of the breast association between transillumination breast spectroscopy and quantitative mammographic features of the breast", Cancer Epidemiol. Biomarkers Prev. 17, 1043 (2008). doi: 10.1158/1055-9965.EPI-07-2658

24. B. Pogue, S. Jiang, H. Dehghani, C. Kogel, S. Soho, S. Srinivasan, X. Song, T.D. Tosteson, S.P. Poplack and K.D. 
Paulsen, "Characterization of hemoglobin, water, and NIR scattering in breast tissue: analysis of intersubject variability and menstrual cycle changes", J. Biomed. Opt. 9, 541 (2004). doi: 10.1117/1.1691028

25. M. Simick, R. Jong, B. Wilson and L. Lilge, “Nonionizing nearinfrared radiation transillumination spectroscopy for breast tissue density and assessment of breast cancer risk," J. Biomed. Opt. 9, 794 (2004). doi: 10.1117/1.1758269

26. L. Spinelli, “Bulk optical properties and tissue components in the female breast from multiwavelength timeresolved optical mammography", J. Biomed. Opt. 9, 1137 (2004). doi: $\underline{10.1117 / 1.1803546}$

27. W. Becker, Advanced Time-Correlated Single Photon Counting Techniques. Springer Verlag, Berlin, Germany (2005). doi: $10.1007 / 3-540-28882-1$

28. A. Pifferi, A. Torricelli, P. Taroni, D. Comelli, A. Bassi and R. Cubeddu, "Fully automated time domain spectrometer for the absorption and scattering characterization of diffusive media", Rev. Sci. Instrum. 78, 053103 (2007). doi: $10.1063 / 1.2735567$

29. W.H. Press, S.A. Teukolsky, W.T. Vetterling and B.P. Flannery, Numerical Recipes in C: The Art of Scientific Computing. Cambridge University Press, New York, USA (1992).

30. S. Prahl, Oregon Medical Laser Center website. Available at: (http://omlc.ogi.edu/spectra)

31. Biomedical Optics Research Laboratory (BORL) at UCL (2005). Available at: (http://www.medphys.ucl.ac.uk/ research/borl/research/NIR topics/spectra/spectra. $\underline{\text { html }}$

32. P. Taroni, A. Bassi, D. Comelli, A. Farina, R. Cubeddu and A. Pifferi, "Diffuse optical spectroscopy of breast tissue extended to $1100 \mathrm{~nm}$ ", J. Biomed. Opt. 14, 054030 (2009). doi: $\underline{10.1117 / 1.3251051}$

33. J.T. Kuenstner and K.H. Norris, "Spectrophotometry of human haemoglobin in the near infrared region from 1000 to 2500 nm", J. Near Infrared Spectrosc. 2, 484 (1994). doi: 10.1255/jnirs.32

34. J.T. Kuenstner and K.H. Norris, "Near infrared haemoglobinometry", J. Near Infrared Spectrosc. 3, 11 (1995). doi: $10.1255 /$ jnirs. 50

35. L. Kou, D. Labrie and P. Chylek, "Refractive indices of water and ice in the $0.652 .5 \mu \mathrm{m}$ spectral range", Appl. Opt. 32, 3531 (1993). doi: 10.1364/A0.32.003531

36. G.M. Hale and M.R. Querry, "Optical constants of water in the $200 \mathrm{~nm}$ to $200 \mu \mathrm{m}$ wavelength region", Appl. Opt. 12, 555 (1973). doi: 10.1364/A0.12.000555

37. S. Matcher, M. Cope and D. Delpy, "Use of the water absorption spectrum to quantify tissue chromophore concentration changes in near infrared spectroscopy", Phys. Med. Biol. 39, 177 (1994). doi: 10.1088/00319155/39/1/011

38. S.H. Chung, A.E. Cerussi, C. Klifa, H.M. Baek, O. Birgul, G. Gulsen, S.I. Merritt, D. Hsiang and B.J. Tromberg, "In vivo water state measurements in breast cancer using broadband diffuse optical spectroscopy", Phys. Med. Biol. 53, 6713 (2008). doi: 10.1088/0031-9155/53/23/005

39. S. Kukreti, A. Cerussi, B. Tromberg and E. Gratton, “Intrinsic tumor biomarkers spectroscopic analysis of near-infrared spectra”, J. Biomed. Opt. 12, 020509 (2007). doi: $10.1117 / 1.2709701$

40. R.L.P. van Veen, H.J.C.M. Sterenborg, A. Pifferi, A. Torricelli, E. Chikoidze and R. Cubeddu, “Determination of visible near-IR absorption coefficients of mammalian fat using time- and spatially resolved diffuse reflectance and transmission spectroscopy", J. Biomed. Opt. 10, 054004 (2005). doi: 10.1117/1.2085149

41. "Bovine Collagen Type I", (www.sigma-aldricht.com)

42. J.R. Mourant, T. Fuselier, J. Boyer, T.M. Johnson and I.J. Bigio, "Predictions and measurements of scattering and absorption over broad wavelength ranges in tissue phantoms", Appl. Opt. 36, 949 (1997). doi: $10.1364 /$ A0.36.000949

43. A.M.K. Nilsson, C. Sturesson, D.L. Liu and S. Andersson-engels, "Changes in spectral shape of tissue optical properties in conjunction with laser-induced thermotherapy", Appl. Opt. 37, 1256 (1998). doi: $10.1364 /$ A0.37.001256

44. C. D’Andrea, L. Spinelli, A. Bassi, A. Giusto, D. Contini, J. Swartling, A. Torricelli and R. Cubeddu, "Time-resolved spectrally constrained method for the quantification of chromophore concentrations and scattering parameters in diffusing media", Opt. Expr. 14, 1888 (2006). doi: 10.1364/OE.14.001888

45. F. Martelli, S. Del Bianco, A. Ismaelli and G. Zaccanti, Light Propagation through Biological Tissue and Other Diffusive Media: Theory, Solutions, and Software. SPIE Press Group, Bellingham, USA (2009).

46. A. Tosi, A. Dalla Mora, F. Zappa and S. Cova, "Singlephoton avalanche diodes for the near-infrared range: detector and circuit issues", J. Mod. Opt. 56, 299 (2009). doi: 10.1080/09500340802263075

47. A. Pifferi, J. Swartling, E. Chikoidze, A. Torricelli, P. Taroni, A. Bassi, S. Andersson-Engels and R. Cubeddu, “Spectroscopic time-resolved diffuse reflectance and transmittance measurements of the female breast at different interfiber distances", J. Biomed. Opt. 9, 1143 (2004). doi: 10.1117/1.1802171

48. R. Cubeddu, C. D’Andrea, A. Pifferi, P. Taroni, A. Torricelli and G. Valentini, "Effects of the menstrual cycle on the red and near-infrared optical properties of the human breast", Photochem. Photobiol. 72, 383 (2000).

49. P.M. Vogel, N.G. Georgiade, B.F. Fetter, F.S. Vogel and K.S. McCarty, "The correlation of histologic changes in the human breast with the menstrual cycle", Am. J. Pathol. 104, 23 (1981).

50. V.A. McCormack and I. dos Santos Silva, "Breast density and parenchymal patterns as markers of breast cancer risk: A meta-analysis", Cancer Epidemiol. Biomarkers Prev. 15, 1159 (2006). doi: 10.1158/1055-9965.EPI-06$\underline{0034}$ 
51. K. Kerlikowske and A.I. Phipps, "Breast density influences tumor subtypes and tumor aggressiveness", J. Natl. Cancer Inst. 103, 1143 (2011). doi: 10.1093/jnci/ djr263

52. L. Yaghjyan, G. Colditz, L. Collins, S. J. Schnitt, B. Rosner, C. Vachon and R.M. Tamimi, "Mammographic breast density and subsequent risk of breast cancer in postmenopausal women according to tumor characteristics", J. Natl. Cancer Inst. 103, 1179 (2011). doi: 10.1093/jnci/ djr225

53. J. Couzin, "Dissecting a hidden breast cancer risk”, Science 309, 1664 (2005). doi: 10.1126/science.309.5741.1664

54. P.P. Provenzano, D.R. Inman, K.W. Eliceiri, J.G. Knittel, L. Yan, C.T. Rueden, J.G. White and P.J. Keely, "Collagen density promotes mammary tumor initiation and progression", BMC Med. 6, 11 (2008). doi: 10.1186/17417015-6-11
55. Y.-P. Guo, L.J. Martin, W. Hanna, D. Banerjee, N. Miller, E. Fishell, R. Khokha and N.F. Boyd, "Growth factors and stromal matrix proteins associated with mammographic densities growth factors and stromal matrix proteins associated with mammographic densities", Cancer Epidemiol. Biomarkers Prev. 10, 243 (2001).

56. S.A. Carp, J. Selb, Q. Fang, R. Moore, D.B. Kopans, E. Rafferty and D.A. Boas, "Dynamic functional and mechanical response of breast tissue to compression", Opt. Expr. 16, 16064 (2008)

57. A. Pifferi, A. Torricelli, L. Spinelli, D. Contini, R. Cubeddu, F. Martelli, G. Zaccanti, A. Tosi, A. Dalla Mora, F. Zappa and S. Cova, "Time-resolved diffuse reflectance using small source-detector separation and fast single-photon gating," Phys. Rev. Lett. 100, 138101 (2008). doi: 10.1103/ PhysRevLett.100.138101 
